TM-1585

\title{
Calculation of Beam Sweeping Effect for the Fermilab Antiproton Source
}

\author{
C. M. Bhat and N. V. Mokhov* \\ Fermi National Accelerator Laboratory \\ P.O. Box 500, Batavia, Illinois \\ *Permanent address: \\ Institute for High Energy Physics \\ Serpukhov, U.S.S.R.
}

April 5, 1989 


\title{
Calculation of Beam Sweeping Effect for the Fermilab Antiproton Source
}

\author{
C.M.Bhat and N.V.Mokhov* \\ Fermi National Accelerator Laboratory \\ P.O. Box 500, Batavia, Illinois 60510 \\ * Permanent address: \\ Institute for High Energy Physics \\ Serpukhov, U.S.S.R.
}

April 5, 1989

\section{Introduction}

One of the goals in the Tevatron upgrade is to achieve luminosity beyond $L=10^{31} \mathrm{~cm}^{-2} \mathrm{sec}^{-1}$. This is done by increasing the intensity and the phase space density of both proton and antiproton beams and/or by decreasing average beam spot sizes[1]. For this, the antiproton source should be capable of producing high intensity and low emittance antiproton beam in a relatively short time compared to the present average pbar stack rate of about 1.2-1.6 $m A /$ hour. The antiprotons are produced by bombarding $120 \mathrm{GeV}$ protons directly on to a thick target. Therefore, in the first place, the pbar yield could be increased merely by increasing number of protons in a bunch. However, it is believed that currently the target is very close to its high temperature limits: melting and cracking due to shock waves creation $[2,3]$.

In the previous collider run the target materials used were heavymet (an alloy of $90 \% \mathrm{~W}, 6 \% \mathrm{Cu}$ and $4 \% \mathrm{Ni}$ ), copper and tantalum. A study of the irradiated targets[4] has shown results in a good agreement with recent theoretical investigations[3], which predict allowable number of protons in a 
pulse on tungsten to be

$$
N_{p} \simeq 2 \times 10^{12} \sigma .
$$

Here $\sigma$ is R.M.S. beam spot size in $m m$.

Eq.(1) corresponds to a maximum energy density in the target equal to 200 Joules/gm (a shock wave limit for tungsten[1]). Using the same limit for copper at the current antiproton source parameters one obtaines $N_{p}$, which is at least a factor of three larger. Moreover, recent observations at Fermilab are indicating that a shock wave limit in copper very likely could be as high as $600 \mathrm{Joules} / \mathrm{gm}$, i.e., an additional factor of three in $N_{p}$. The appropriate thickness of copper target is comparable to the tungsten one. All of that, together with almost the same antiproton yield from copper and tungsten targets[3], leads to a conclusion that copper is the most suitable target material for pbar production.

Thus the tolerable number of protons on a target grows as the beam size increases. On the other hand, to get a high phase space density of the produced pbars within a given angular acceptance one needs to decrease the proton beam spot size at the upstream of target as much as possible[1].

The above difficulty can be overcome by sweeping the proton beam on the target using horizontal and vertical beam kickers in the beam line at pbar production target station. Below we present an estimation of reduction in deposited energy density in the target by sweeping the proton beam circularly with a fixed radius. The beam sweeping scheme is described in section 2 . Section 3 deals with an analytical approach for a very thin target. Detailed Monte Carlo calculations for thick target are given in section 4 .

\section{Beam sweeping scheme}

The most feasible sweeping system may be displacing the beam on the target in a circular path with a fixed radius. This can be achieved by means of two magnets placed upstream of the target and excited by a sinusodial current with the phase difference between these magnet currents of $\pi / 2$. The beam sweeping has to be synchronized with main ring beam pulse. The period of oscillation of sinusodial current should be exactly same as beam spill time $(1.6 \mu \mathrm{sec})$. Another set of kicker magnets matched for $8.9 \mathrm{GeV} / \mathrm{c}$ antiprotons with momentum acceptance of about $4 \%$ is placed downstream of the target 
after the focusing Li-lens to bring the beam back to its normal path. To avoid accidental interaction of non-sweeping high intensity proton beam with the target an appropriate beam abort system has to be provided. Details of possible beam sweeping systems which can be adopted at AP0 target station have been discussed in $[5,6]$.

\section{Possible gain from beam sweeping}

To estimate the energy density reduction by beam sweeping let us consider at first a thin target. The dominant mechanism of the energy deposition during the interaction of high energy protons with a very thin layer of matter is by ionization of the medium. Other processes, nuclear de-excitation and electromagnetic showers, contribute to less than $20 \%$ at depth less than a few millimeters of medium[3,7]. Therefore, to a first approximation, the energy deposition density for such a target can be written as

$$
\epsilon(r)=\frac{d E}{d x} \times \Phi(r),
$$

where $\Phi(r)$ is a particle fluence and $d E / d x$ is the ionization loss rate ( $\simeq$ $1.9 \times 10^{-3} \mathrm{GeV} \times \mathrm{cm}^{2} / \mathrm{gm}$ for copper).

For practical purposes $\Phi(r)$ can be assumed to be the incident proton flux. Let us suppose the beam is along $z$-axis and beam sweeping is parallel to $z$-axis. Depending upon type of beam distribution two cases can be considered: uniform flux and Gaussian-like flux.

\subsection{Uniform flux}

For the reference, no sweeping case the uniform distribution of flux at a target face in the restricted circular area of radius $\sigma$ is given by

$$
\Phi(r)=\frac{1}{\pi \sigma^{2}}
$$

For the same uniform beam of radius $\sigma$ swept over an anular region of radius $R \geq \sigma$ the flux is represented by

$$
\Phi_{s}(r)=\frac{1}{\pi\left((R+\sigma)^{2}-(R-\sigma)^{2}\right)}=\frac{1}{4 \pi \sigma R} .
$$


Using (2-4) one can get the gain of sweeping

$$
\frac{\epsilon}{\epsilon_{s}}=\frac{4 R}{\sigma}
$$

which is equal to 10 for typical parameters $R=0.025 \mathrm{~cm}, \sigma=0.01 \mathrm{~cm}$.

\subsection{Gaussian-like tlux}

Let us assume that beam has a Gaussian distribution with a standard deviation of $\sigma$. If such a beam is centered at $z$-axis, then flux is given by

$$
\Phi(r)=\frac{A}{2 \pi \sigma^{2}} \exp \left(-\frac{r^{2}}{2 \sigma^{2}}\right),
$$

with $A=1$.

For beam sweeping with radius $R$

$$
\Phi_{s}(r)=\frac{A_{a}}{2 \pi \sigma^{2}} \exp \left(-\frac{(r-R)^{2}}{2 \sigma^{2}}\right)
$$

with $A_{s}$ dependent on sweeping radius

$$
A_{\mathbf{s}}=\frac{\sigma^{2}}{\int_{0}^{\infty} \exp \left(-\frac{(r-R)^{2}}{2 \sigma^{2}}\right) r d r}
$$

Here one can notice that the uniform energy density defined by (3) is exactly twice that of the Gaussian peak density $\epsilon_{\max }=d E / d x \times 1 /\left(2 \pi \sigma^{2}\right)$.

\subsection{Numerical data}

Using Eqs. (2-8) the maximum energy density in a target has been estimated. Table 1 gives a comparison of results for uniform and Gaussian beams of 120 $\mathrm{GeV}$ protons irradiating a thin copper target. Data are given for various $\sigma$ and for both cases: axial non-sweeping beam and sweeping beam of sweeping radius $R=0.025 \mathrm{~cm}$. In the last column the corresponding results of Monte Carlo calculations with MARS10 program[7,8] are presented for a 1 cm copper target. Statistical errors are about $5 \%$.

Generally the data agree very well. Eqs. (7-8) underestimate the result only for sweeping case with $\sigma=R$. From the table 1 it is clear that using 
a proton beam sweeping system with $R / \sigma>1$ one can achieve a significant reduction of energy deposition density.

Table 1 : Maximum energy density in a thin target (GeV/gm per proton)

\begin{tabular}{ccccc}
\hline$\sigma(\mathrm{cm})$ & Sweeping & Uniform beam & Gaussian beam & Gaussian (MARS10) \\
\hline 0.005 & No & 24.20 & 12.10 & 10.00 \\
& Yes & 1.21 & 0.98 & 0.97 \\
\hline 0.015 & No & 2.68 & 1.34 & 1.30 \\
& Yes & 0.40 & 0.31 & 0.42 \\
\hline 0.025 & No & 0.97 & 0.48 & 0.49 \\
& Yes & 0.25 & 0.18 & 0.32 \\
\hline
\end{tabular}

\section{Monte Carlo results}

The pbar targets are generally several centimeters thick and hence a more rigorous estimation of energy density depletion by a beam sweeping on the target has to be made. We have performed extensive Monte Carlo calculations of hadronic cascades in the copper target using MARS10 program[7,8]. All the calculations have been made for cylindrical geometry and assuming target length of $7 \mathrm{~cm}$.

Results on the maximum energy deposition density in the target which occurs at the downstream end $(z \simeq 5-7 \mathrm{~cm})$ are presented in Fig. 1 through Fig.3 for beams with and without sweeping as function of sweeping radius $R$ and beam RMS spot size $\sigma$. It turns out that the effect of sweeping is seen only if $\sigma \leq R$ and becomes important for small values of $\sigma / R$. It is remarkable that results are in a qualitative and quantitative agreement with the predictions of Sect.3.

Fig.4 shows lateral distributions of energy density at the upstream and downstream ends of the target for $\sigma=0.01 \mathrm{~cm}$. As one could expect, the distributions in the sweeping case are much more uniform compared to the reference case. A maximum is reached at a radius equal to the sweeping radius $R$ (that is true even for $R \sim 0$ ).

The calculated maximum temperature in the target irradiated with a beam of $1.5 \times 10^{12}$ protons and $\sigma=0.01 \mathrm{~cm}$ is shown in Fig.5 as function of 
the sweeping radius $R$. By properly choosing $R$ one can keep the maximum temperature in the target well below the melting point (for copper it is 1356 $\left.{ }^{0} K\right)$.

It may be interesting to note that at the present operating conditions (no beam sweeping, $1.73 \times 10^{12}$ protons per pulse and $\sigma=0.018 \mathrm{~cm}$ ) the antiproton source is almost at its limit, because the corresponding maximum energy deposition in copper target is very close to the melting point.

By decreasing $\sigma$ from $0.018 \mathrm{~cm}$ to $0.01 \mathrm{~cm}$ and sweeping beam on the target circulary with radius $R \simeq 0.025 \mathrm{~cm}$, the maximum energy deposited could be decreased by about a factor of three and correspondingly the beam intensity can be increased to the same quantity. At the same time antiproton phase space could be increased by about $15 \%$.

\section{Conclusions}

The problems arising from targeting high intensity small- $\sigma$ proton pulses for antiproton production can be reduced by the beam sweeping on the target. The presented results of analytical estimations and of the full-scale MonteCarlo studies indicate a significant efficiency of diluting the beam energy deposition. These results are useful to obtain optimum design parameters for various magnetic elements to be put in at the upstream end of the target to develop a beam sweeping system.

The authors are sincerely grateful to G.Dugan and J.Marriner for helpful comments. 


\section{References}

[1] Design Report TEVATRON1 Project, Fermilab (1984).

[2] C. Hojvat and A. Van Ginneken, Nucl. Instr. and Methods 206, 67 (1983).

[3] I. L. Azhgirey and N. V. Mokhov, Fermilab TM-1529 (1988).

[4] M. Gormley, Private communication.

[5] F. Krienen and F. Mills, Fermilab PBAR Note-70 (1980).

[6] T. Vsevolozhskaya et al, Fermilab TM-1048 (1981).

[7] N. V. Mokhov, Sov. J. Part. Nucl. 18(5), 408 (1987).

[8] N. V. Mokhov, Fermilab FN-509 (1989). 


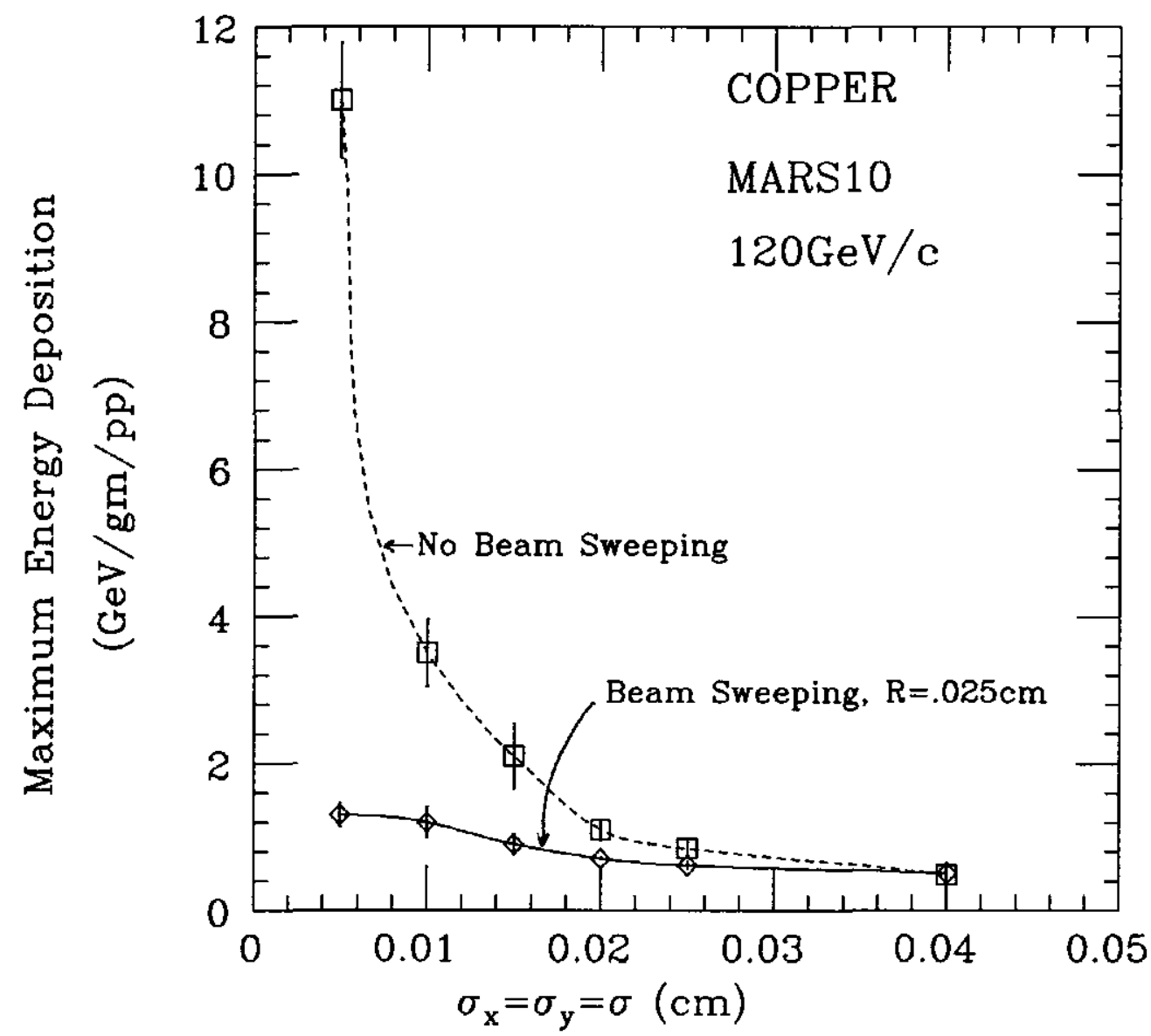

Fig.1 Maximum energy deposition versus average beam spot size for beam sweeping $(R=0.025 \mathrm{~cm})$ and no beam sweeping. Curves are drawn to guide the eye. Vertical bars represent statistical errors. 


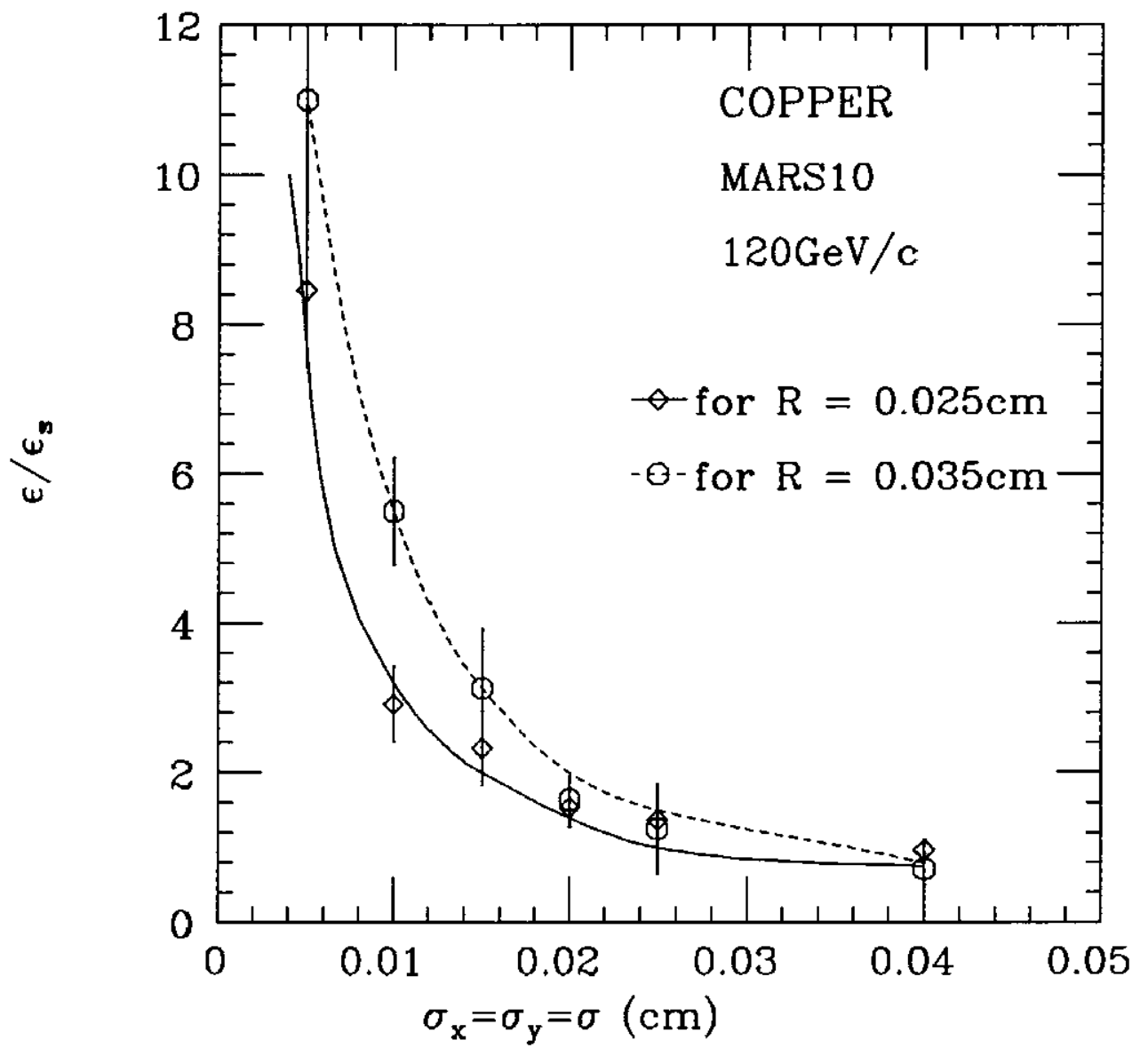

Fig.2. The ratio of maximum energy deposited for no beam sweeping to beam sweeping case versus beam spot size. Curves are drawn to guide the eye. 


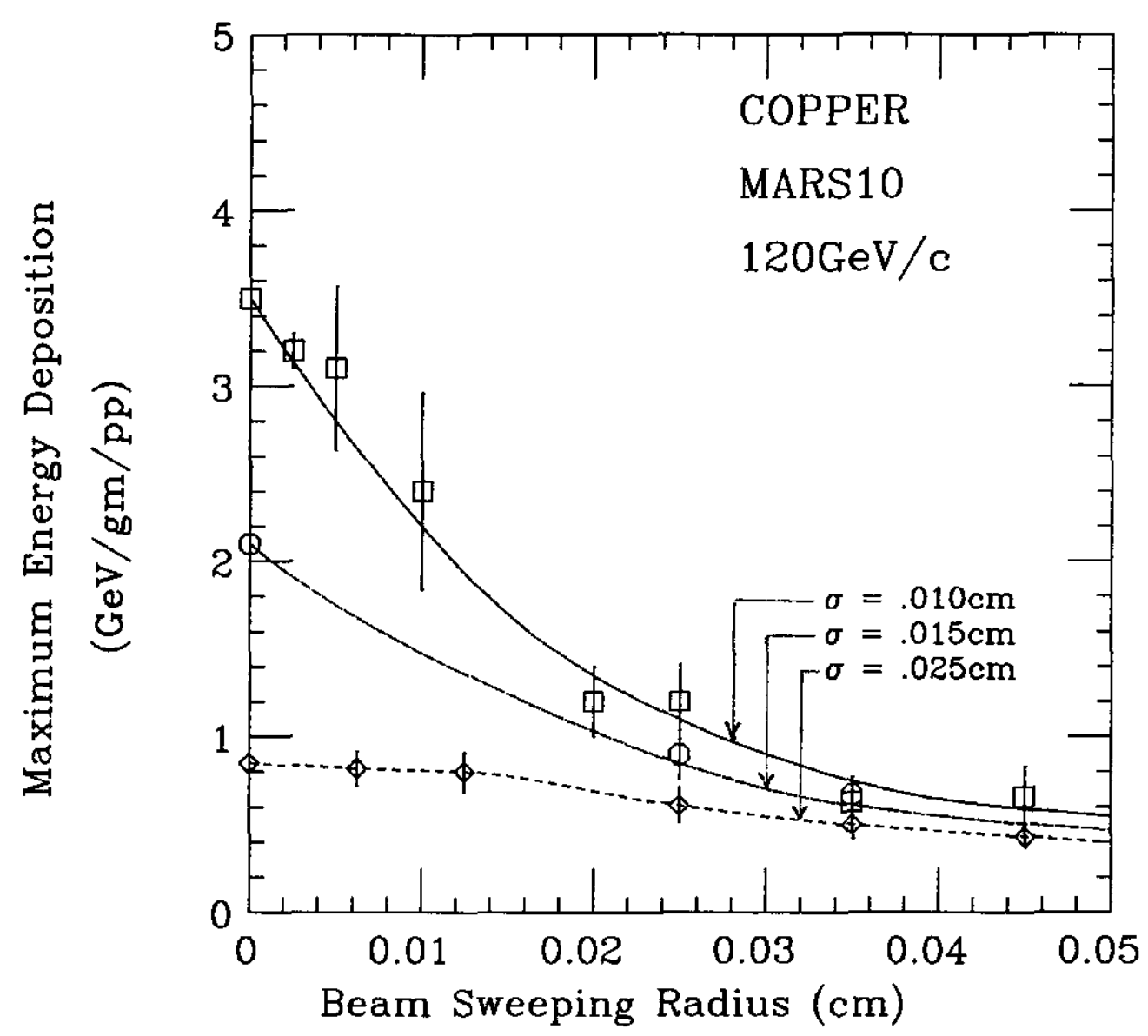

Fig.3. Maximum energy deposited versus beam sweeping radius for beam spot sizes $0.010,0.015$ and $0.025 \mathrm{~cm}$. Errors are purely statistical. 


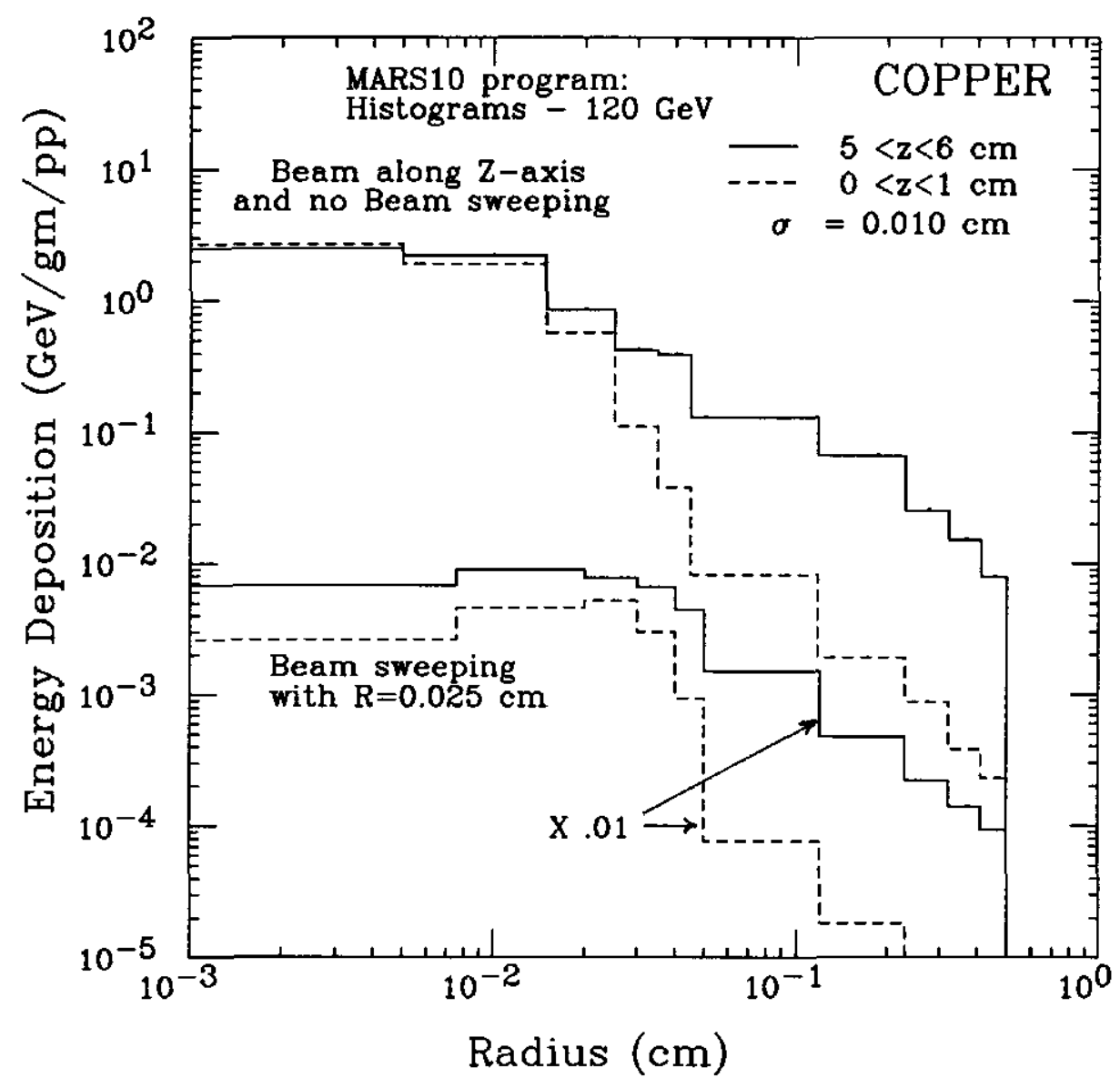

Fig.4. Radial distribution of energy deposition at down stream and upstream ends of the target for beam sweeping and no beam sweeping cases. 


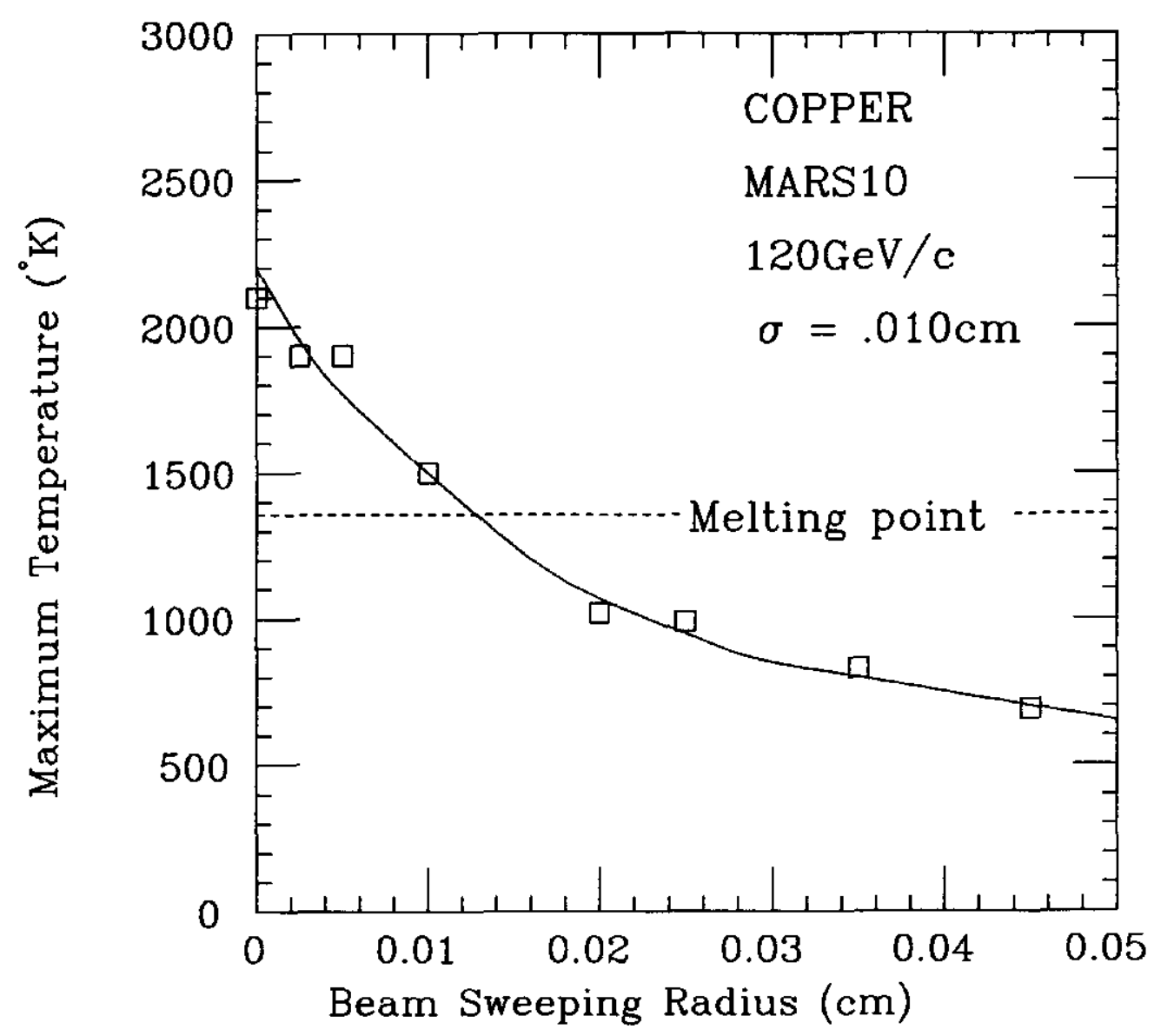

Fig.5. Maximum temperature in the target as a function of the beam sweeping radius for beam spot size $0.010 \mathrm{~cm}$. 